\title{
Insuficiencia renal aguda secundaria al tratamiento con Aciclovir por herpes zoster en pacientes con lupus eritematoso sistémico: reporte de dos casos
}

\author{
Marcos Reque ${ }^{1,2,3}$, Emma Mariana Ábalos ${ }^{3}$ \\ ${ }^{1}$ Hospital General de Agudos José María Ramos Mejía. Buenos Aires. Argentina \\ ${ }^{2}$ Hospital Naval Cirujano Mayor Dr. Pedro Mallo. Buenos Aires. Argentina \\ ${ }^{3}$ Instituto Superior de Enfermería Artémides Zatti. Argentina
}

Como citar este artículo:

Reque M, Ábalos EM.

Insuficiencia renal aguda secundaria al tratamiento con Aciclovir por herpes zoster en pacientes con lupus eritematoso sistémico: reporte de dos casos.

Enferm Nefrol. 2021 Oct-Dic;24(4):423-30.

Se presentan dos casos de pacientes con lupus eritematoso sistémico con recidiva de herpes zoster, que desarrollan insuficiencia renal aguda secundaria al tratamiento con Aciclovir endovenoso.

Descripción del plan de cuidados: El plan de cuidados estuvo abocado a tratar las necesidades alteradas según la clasificación de Virginia Henderson. Estas necesidades alteradas fueron: la necesidad de eliminación, higiene e integridad de la piel, dolor y movilización. Para el proceso de atención de enfermería se aplicó la taxonomía NANDA.

Evaluación del plan: Tras aplicar el proceso de atención de enfermería se logró mejorar la función renal, recuperar la integridad cutánea, se evitó infecciones por las lesiones en la piel producidas por el herpes zoster, se controló el dolor, y se mejoró la movilidad del brazo izquierdo de una de las pacientes que padecía paresia.

Conclusiones: Es menester para enfermería realizar un seguimiento minucioso de los pacientes que presentan tratamiento con Aciclovir, especialmente de aquellos

\section{Correspondencia:}

Emma Mariana Abalos

E-mail: inte@hotmail.com.ar que presentan patologías crónicas de base, como lupus eritematoso sistémico en recidivas con herpes zoster, para prevenir eventos adversos asociados al fármaco.

PALABRAS CLAVE: herpes zóster; aciclovir; lupus sistémico eritematoso; efecto adverso; cuidados de enfermería.

Acute renal failure secondary to Acyclovir treatment for herpes zoster in patients with systemic lupus erythematosus: report of two cases

Case description: Two cases of patients with systemic lupus erythematosus with herpes zoster recurrence, who develop acute renal failure secondary to treatment with intravenous acyclovir, have been studied.

Description of the care plan: The care plan was aimed at addressing the altered needs according to the Virginia Henderson classification. These altered needs were the need for elimination, hygiene and skin integrity, pain and mobilisation. The NANDA taxonomy was applied for the nursing care process.

Evaluation of the plan: After applying the nursing care process, renal function was improved, skin integrity was 
restored, infections were avoided due to skin lesions caused by herpes zoster, pain was controlled, and the mobility of the left arm of one of the patients suffering from paresis was improved.

Conclusions: Close monitoring by nursing staff of patients treated with acyclovir is necessary, especially those with chronic underlying pathologies, such as systemic lupus erythematosus in relapses with herpes zoster, in order to prevent adverse events associated with the drug.

KEYWORDS: herpes zoster; acyclovir; systemic lupus erythematosus; adverse effect; nursing care.

\section{Introducción}

El Aciclovir es un fármaco antiviral de primera elección en el tratamiento de herpes simple y herpes zoster. Los efectos secundarios más frecuentes producidos por el fármaco son intolerancia gastrointestinal, flebitis, neurotoxicidad y nefrotoxicidad entre otras ${ }^{1}$.

La nefrotoxicidad es el evento adverso más grave que se ha reportado como consecuencia del tratamiento con Aciclovir ${ }^{1}$. La monitorización de la función renal es fundamental para detectar estos casos y prevenirlos, en especial en aquellos pacientes que presentan patologías crónicas como el lupus eritematoso sistémico (LES)².

El riñón es el órgano más importante en el proceso de eliminación de los fármacos por lo que los riñones son susceptibles a la lesión inducida por estos ${ }^{3}$. Se han descrito varios casos de insuficiencia renal aguda (IRA) como efectos secundarios a tratamientos medicamentosos como Aciclovir, sulfonamidas, metotrexato, entre otros. El mecanismo fisiopatológico se relaciona con la cristalización del fármaco en los túbulos renales debido a su insolubilidad en la orina, por lo que estos, al depositarse en el parénquima renal producen daño especialmente en el túbulo e intersticio, desarrollando nefropatía por depósito de cristales ${ }^{4,5}$. La nefritis intersticial se produce por la hipersensibilidad al fármaco. La necrosis tubular aguda es un proceso dosis-dependiente que se desarrolla por toxicidad directa sobre el epitelio tubular. La presentación clínica de dicha nefropatía puede ser aguda o crónica y puede derivar en insuficiencia renal'.

A continuación, se presentarán dos casos clínicos que ocurrieron en el año 2017 en el Hospital Ramos Mejía de la Provincia de Buenos Aires, Argentina.

\section{Caso clínico $\mathrm{N}^{\circ} 1$}

Mujer de 35 años que acude a urgencias por herpes zoster. Se decide su ingreso hospitalario para tratamiento con Aciclovir endovenoso.

Antecedentes clínicos: la paciente presenta como patología de base LES, diagnosticado en 2006, nefritis lúpica grado IV e hipertensión arterial, en tratamiento con betabloqueantes. La paciente afirma no tener antecedentes quirúrgicos, alergias medicamentosas ni consumo de otros medicamentos o sustancias tóxicas.

El tratamiento médico indicado incluyó: dieta hiposódica, tratamiento antiviral con Aciclovir 1 gr endovenoso cada 8 horas, y se continuó con medicación de base antihipertensiva. Durante los dos primeros días de tratamiento con antiviral los valores de laboratorio cambiaron drásticamente. A fecha de ingreso el paciente presentaba un valor de urea de $22 \mathrm{mg} / \mathrm{dl}$ y creatinina $0,5 \mathrm{mg} / \mathrm{dl}$, estos valores fueron aumentando paulatinamente, presentando el primer día de tratamiento urea $48 \mathrm{mg} / \mathrm{dl}$, creatinina $1,85 \mathrm{mg} / \mathrm{dl}$, el segundo día de tratamiento, urea $62 \mathrm{mg} / \mathrm{dl}$, creatinina 7,78 $\mathrm{mg} / \mathrm{dl}$, alcanzando el tercer día urea $69 \mathrm{mg} / \mathrm{dl}$ y creatinina $3,40 \mathrm{mg} /$ dl. La paciente presentó progresión a IRA, apareciendo hiperfosfatemia e hipomagnesemia.

Tras la suspensión del antiviral la urea y creatinina comenzaron a descender lentamente, alcanzando a las 48 horas valores de $57 \mathrm{mg} / \mathrm{dl}$ y $1,38 \mathrm{mg} / \mathrm{dl}$ respectivamente. Los balances hídricos fueron neutros, con mejora progresiva de la función renal.

\section{Caso clínico $\mathrm{n}^{\circ} 2$}

Mujer de 67 años, que acude al hospital por presentar lesión eritematosa compatible con herpes zoster, por lo que se decide su ingreso hospitalario para tratamiento con Aciclovir endovenoso.

Antecedentes clínicos: la paciente presentaba como patología de base LES, diabetes mellitus tipo 2, tratada con medicación hipoglucemiante previo a almuerzo y cena, esclerodermia diagnosticada en el año 2000. Afirmaba no tener alergias medicamentosas ni consumo de otros medicamentos o sustancias tóxicas.

El tratamiento médico indicado incluyó: dieta hipocalórica, tratamiento antiviral con Aciclovir 1 gr, endovenoso cada 8 horas y se continuó con medicación de base. Tras 8 días de tratamiento con Aciclovir la paciente evolucionó con paresia braquial izquierda. Se realizó tomo- 
grafía cerebral sin particularidades y punción lumbar, siendo el líquido cefalorraquídeo: limpio e incoloro, glucorraquia de $46 \mathrm{mg} / \mathrm{dl}$, proteinorraquia $98 \mathrm{mg} / \mathrm{dl}$, leucocitos $0-1 \mathrm{~mm}$. PCR virales negativas. Se constataron un aumento de los valores de laboratorios sanguíneos de urea $(42 \mathrm{mg} / \mathrm{dl})$ y creatinina $(1,19 \mathrm{mg} / \mathrm{dl})$ con progresión a una IRA, por lo que se decidió suspender el tratamiento con Aciclovir.

\section{Proceso de atención de enfermería (PAE).}

En ambos casos se realizó la valoración de enfermería utilizando el modelo de 14 necesidades de Virginia Henderson (ver tabla 1).

Se realizó el proceso de atención de enfermería, priorizando las necesidades más urgentes de atender. El plan

Tabla 1. Valoración Según Virginia Henderson.

\begin{tabular}{|c|c|c|c|}
\hline $\mathbf{N}$ & 14 necesidades & Caso Clínico n 1 & Caso Clínico $n^{0} 2$ \\
\hline 1 & Respiración & $\begin{array}{l}\text { N/A: Tensión arterial: } 130 / 80 \mathrm{mmHg} \text {; fre- } \\
\text { cuencia respiratoria: } 16 \text { respiraciones por } \\
\text { min; frecuencia cardíaca: } 91 \text { latidos por min; } \\
\text { Saturación de } 0_{2}: 98 \% \text {. }\end{array}$ & $\begin{array}{l}\text { N/A: Tensión arterial: } 110 / 70 \mathrm{mmHg} \text {; fre- } \\
\text { cuencia respiratoria: } 20 \text { respiraciones por } \\
\text { min; frecuencia cardíaca: } 84 \text { latidos por min; } \\
\text { Saturación de } 0_{2}: 94 \% \text {. }\end{array}$ \\
\hline 2 & Alimentación/hidratación & $\begin{array}{l}\text { N/A: Buenas condiciones generales, hidratado, } \\
\text { peso: } 64 \mathrm{~kg} \text {. Habitualmente consume dieta } \\
\text { hiposódica. No presenta náuseas y ni vómitos }\end{array}$ & $\begin{array}{l}\text { N/A: Buenas condiciones generales, hidra- } \\
\text { tado, peso: } 88 \mathrm{~kg} \text {. Habitualmente consume } \\
\text { dieta para diabético. No presenta náuseas ni } \\
\text { vómitos. }\end{array}$ \\
\hline 3 & Eliminación & $\begin{array}{l}\text { A: Deterioro de la función renal con aumento } \\
\text { de urea y creatinina hiperfosfatemia e hipo- } \\
\text { magnesemia. } \\
\text { Hábito intestinal adecuado, escala de Bristol } \\
\text { tipo } 3 .\end{array}$ & $\begin{array}{l}\text { A: Deterioro de la función renal con aumento } \\
\text { de urea y creatinina. } \\
\text { Hábito intestinal adecuado, escala de Bristol } \\
\text { tipo } 4 .\end{array}$ \\
\hline 4 & Movilización & $\begin{array}{l}\text { N/A: Deambula por sus propios medios sin } \\
\text { necesidad de ayuda. Paciente diestra. }\end{array}$ & $\begin{array}{l}\text { A: Deambula por sus propios medios sin nece- } \\
\text { sidad de ayuda, pero presenta paresia braquial } \\
\text { izquierda. Paciente diestra. }\end{array}$ \\
\hline 5 & Reposos y sueño & N/A: Puede conciliar el sueño sin dificultad. & N/A: Puede conciliar el sueño sin dificultad. \\
\hline 6 & Vestirse/ desvestirse & N/A: Puede vestirse sin necesidad de ayuda. & N/A: Afebril, temperatura: $36,1^{\circ} \mathrm{C}$. \\
\hline 7 & Termorregulación & N/A: Afebril, temperatura: $36,6^{\circ} \mathrm{C}$. & N/A: Afebril, temperatura: $36,1^{\circ} \mathrm{C}$. \\
\hline 8 & $\begin{array}{l}\text { Higiene e integridad de la } \\
\text { piel }\end{array}$ & $\begin{array}{l}\text { A: Presenta placa eritematosa con abundantes } \\
\text { vesículas y costras en región dorsal desde D3 a } \\
\text { D7 con extensión a región mamaria izquierda } \\
\text { y esternón de } 4 \mathrm{~cm}^{2} \text {, acompañada de eritemas } \\
\text { con calor y dolor. }\end{array}$ & $\begin{array}{l}\text { A: Presenta múltiples vesículas que se ex- } \\
\text { tiende a lo largo de todo el miembro superior } \\
\text { izquierdo y a nivel de D9 de } 5 \mathrm{~cm}^{2} \text {, acompaña- } \\
\text { da de eritemas con calor y dolor. }\end{array}$ \\
\hline 9 & Seguridad & $\begin{array}{l}\text { N/A: Lúcida ubicada en tiempo y espacio sin } \\
\text { signos de déficit o compromiso neurológico. }\end{array}$ & $\begin{array}{l}\text { N/A: Lúcida ubicada en tiempo y espacio, sin } \\
\text { signos de déficit o compromiso neurológico. }\end{array}$ \\
\hline 10 & Comunicación & N/A: No presenta dificultad en la comunicación. & N/A: No presenta dificultad en la comunicación. \\
\hline 11 & Creencias y valores & N/A. & N/A. \\
\hline 12 & Trabajar/autorrealizarse & N/A. & N/A. \\
\hline 13 & Ocio & N/A. & N/A. \\
\hline 14 & Aprendizaje & N/A. & N/A. \\
\hline
\end{tabular}

Necesidades alteradas (A) / no alteradas (NA) 
de cuidados se elaboró utilizando las taxonomías NANDA para los diagnósticos de enfermería, NOC para los criterios de resultados y NIC para las intervenciones enfermeras (ver tabla 2 y tabla 3 ).
Evaluación: Tras aplicar el PAE las pacientes:

- Mejoraronn los niveles séricos de urea y creatinina, lo cual indica una mejoría de la función renal.

Tabla 2. Proceso de Atención de Enfermería con taxonomía NANDA caso clínico número 1.

\begin{tabular}{|c|c|c|}
\hline NANDA & NOC & NIC \\
\hline $\begin{array}{l}\text { 00016. Deterioro de la eliminación } \\
\text { urinaria. } \\
\text { Características definitorias: } \\
\text { - Retención } \\
\text { Factores relacionados: } \\
\text { - Multicausalidad }\end{array}$ & $\begin{array}{l}\text { 0504. Función renal. } \\
\text { Indicadores: } \\
50402 \text {. Balances de ingesta y diuresis en } 24 \\
\text { horas } \\
50405 \text {. Peso específico de la orina } \\
50406 \text {. Color de la orina } \\
50409 \text {. Electrolitos de la orina } \\
50427 \text {. Aumento de la creatinina sérica. } \\
50426 \text {. Aumento del nitrógeno ureico en } \\
\text { sangre } \\
\text { Escala Likert } 2 \text { (de gravemente comprometido } \\
\text { a no comprometido) } \\
\text { Puntuación basal: } 2 \\
\text { Al alta puntuación: } 5\end{array}$ & $\begin{array}{l}\text { 2000. Manejo de electrolitos. } \\
\text { Actividades: } \\
\text { 200001. Observar si los electrolitos en suero } \\
\text { son anormales. } \\
\text { 200002. Observar si hay manifestaciones de } \\
\text { desequilibrio de electrolitos. } \\
\text { 200003. Mantener un acceso endovenoso } \\
\text { permeable. } \\
\text { 200004. Administrar líquidos según prescrip- } \\
\text { ción. } \\
\text { 200005. Mantener un registro adecuado de } \\
\text { entradas y salidas de líquidos. } \\
\text { 2000010. obtener muestra para análisis de } \\
\text { laboratorio de los niveles de electrolitos. } \\
\text { 2000015. Disponer de una dieta adecua- } \\
\text { da para el desequilibrio de electrolitos del } \\
\text { paciente. }\end{array}$ \\
\hline $\begin{array}{l}\text { 00132. Dolor agudo } \\
\text { Características definitorias: } \\
\text { - Expresa dolor } \\
\text { Factores relacionados: } \\
\text { - Agentes lesivos (biológicos, quími- } \\
\text { cos, físicos, psicológicos) }\end{array}$ & $\begin{array}{l}\text { 2102. Nivel del dolor } \\
\text { Indicadores: } 210201 \text {. Dolor referido. } \\
\text { Escala EVA (de } 0 \text { a 10) } \\
\text { Puntuación basal: } 5 \\
\text { Al alta puntuación:10 }\end{array}$ & $\begin{array}{l}\text { 1400. Manejo del dolor } \\
\text { Actividades: } \\
\text { 140001. Realizar una valoración exhaustiva } \\
\text { del dolor que incluya la localización, caracte- } \\
\text { rísticas, aparición / duración, frecuencia, cali- } \\
\text { dad, intensidad o severidad del dolor y factores } \\
\text { desencadenantes. } \\
\text { 140003. Asegurarse de que el paciente reciba } \\
\text { los cuidados analgésicos correspondientes. } \\
\text { 140007. Determinar el impacto de la experien- } \\
\text { cia del dolor sobre la calidad de vida (sueño, } \\
\text { apetito, actividad, función cognoscitiva, humor, } \\
\text { relaciones, trabajo y responsabilidad de roles). } \\
\text { 140013. Determinar la frecuencia necesaria } \\
\text { para la realización de una valoración de la } \\
\text { comodidad del paciente y poner en práctica un } \\
\text { plan de seguimiento. }\end{array}$ \\
\hline $\begin{array}{l}\text { 00046. Deterioro de la integridad } \\
\text { cutánea. } \\
\text { Características definitorias: } \\
\text { - Alteración de la superficie de la } \\
\text { piel } \\
\text { Factores relacionados: } \\
\text { - Déficit inmunitario }\end{array}$ & $\begin{array}{l}\text { 1101. Integridad tisular: piel y membranas } \\
\text { mucosas. } \\
\text { Indicadores: } 110115 \text {. Lesiones Cutáneas. } \\
\text { Escala Likert } 2 \text { (de gravemente comprometido } \\
\text { a no comprometido) } \\
\text { Puntuación basal:2 } \\
\text { Al alta puntuación:5 }\end{array}$ & $\begin{array}{l}\text { 3660. Cuidados de las heridas. } \\
\text { Actividades. } \\
366003 \text {. Monitorizar las características de la } \\
\text { herida, incluyendo drenaje, color, tamaño y olor. } \\
366004 \text {. Medir el lecho de la herida. } \\
366006 \text {. Limpiar/curar la herida según indi- } \\
\text { cación. } \\
366011 \text {. Aplicar el vendaje adecuado al tipo } \\
\text { de herida. } \\
366015 \text {. Cambiar el apósito según la cantidad } \\
\text { de exudado y drenaje. } \\
366016 \text {. Inspeccionar la herida cada vez que } \\
\text { se realiza el cambio de vendaje. } \\
366030 \text {. Documentar la localización, el tama- } \\
\text { no y el aspecto de la herida. } \\
366017 \text {. Comparar y registra cualquier cambio } \\
\text { producido en la herida. }\end{array}$ \\
\hline
\end{tabular}




\begin{tabular}{|c|c|c|}
\hline NANDA & NOC & NIC \\
\hline $\begin{array}{l}\text { 00195. Riesgo de desequilibrio } \\
\text { electrolítico } \\
\text { Factores relacionados: } \\
\text { Disfunción renal }\end{array}$ & $\begin{array}{l}\text { 60612. Aumento del fósforo } \\
\text { 6609. Disminución del magnesio } \\
\text { Escala Likert (desviación grave del rango } \\
\text { normal hasta sin desviación) } \\
\text { Puntuación basal: } 4 \\
\text { Al alta puntuación:5 }\end{array}$ & $\begin{array}{l}\text { 2020. Monitorización de electrolitos } \\
\text { 202001. Vigilar el nivel sérico de electrolitos } \\
202011 \text {. Observar los electrocardiograma para } \\
\text { ver si hay cambios relacionados con niveles } \\
\text { anormales de magnesio }\end{array}$ \\
\hline $\begin{array}{l}\text { 00004. Riesgo de infección } \\
\text { Factores relacionados: } \\
\text { Alteración de las defensas primarias } \\
\text { (rotura de la piel, traumatismo de } \\
\text { los tejidos, disminución de la acción } \\
\text { ciliar, estasis de los líquidos corpora- } \\
\text { les, cambio del pH de las secreciones, } \\
\text { alteración del peristaltismo) }\end{array}$ & $\begin{array}{l}\text { 1902. Control del riesgo } \\
\text { 190201. Reconoce los factores de riesgo } \\
\text { Escala Likert (de siempre demostrado a nunca } \\
\text { demostrado) } \\
\text { Puntuación basal: } 4 \\
\text { Al alta puntuación:5 }\end{array}$ & $\begin{array}{l}\text { 6540. Control de infección } \\
\text { Actividades: } \\
654001 \text {. Distribuir la superficie correspondien- } \\
\text { te por paciente, según los consejos de los Cen- } \\
\text { tros para el control de enfermedades (CDC) } \\
654002 \text {. Limpiar el ambiente adecuadamente } \\
\text { después de cada uso por parte de los pacientes. } \\
654004 \text {. Aislar a las personas expuestas a } \\
\text { enfermedades transmisibles. } \\
654006 \text {. Limitar el número de visitas } \\
654017 \text {. Fregar la piel del paciente con un } \\
\text { agente antibacteriano. }\end{array}$ \\
\hline
\end{tabular}

Tabla 3. Proceso de Atención de Enfermería con taxonomía NANDA caso clínico n 2.

\begin{tabular}{|c|c|c|}
\hline NANDA & NOC & NIC \\
\hline $\begin{array}{l}\text { 00016. Deterioro de la eliminación } \\
\text { urinaria. } \\
\text { Características definitorias: } \\
\text { - Retención } \\
\text { Factores relacionados: } \\
\text { - Multicausalidad }\end{array}$ & $\begin{array}{l}\text { 0504. Función renal. } \\
\text { Indicadores: } \\
50427 \text {. Aumento de la creatinina } \\
\text { sérica. } \\
50426 \text {. Aumento del nitrógeno ureico } \\
\text { en sangre } \\
\text { Escala Likert } 2 \text { (de gravemente com- } \\
\text { prometido a no comprometido) } \\
\text { Puntuación basal:2 } \\
\text { Al alta puntuación:5 }\end{array}$ & $\begin{array}{l}\text { 2000. Manejo de electrolitos. } \\
\text { Actividades: } \\
200001 \text {. Observar si los electrolitos en suero son } \\
\text { anormales. } \\
\text { 200002. Observar si hay manifestaciones de desequili- } \\
\text { brio de electrolitos. } \\
\text { 200003. Mantener un acceso endovenoso permeable. } \\
\text { 200004. Administrar líquidos según prescripción. } \\
\text { 200005. Mantener un registro adecuado de entradas y } \\
\text { salidas de líquidos. } \\
\text { 2000010. Obtener muestra para análisis de laboratorio } \\
\text { de los niveles de electrolitos. } \\
\text { 2000015. Disponer de una dieta adecuada para el } \\
\text { desequilibrio de electrolitos del paciente }\end{array}$ \\
\hline $\begin{array}{l}\text { 00132. Dolor agudo } \\
\text { Características definitorias: } \\
\text { - Expresa dolor } \\
\text { Factores relacionados: } \\
\text { - Agentes lesivos (biológicos, quími- } \\
\text { cos, físicos, psicológicos) }\end{array}$ & $\begin{array}{l}\text { 2102. Nivel del dolor } \\
\text { Indicadores: } 210201 \text {. Dolor referido. } \\
\text { Escala EVA (de } 0 \text { a 10) } \\
\text { Puntuación basal: } 6 \\
\text { Al alta puntuación:10 }\end{array}$ & $\begin{array}{l}\text { 1400. Manejo del dolor } \\
\text { Actividades: } \\
\text { 140001. Realizar una valoración exhaustiva del dolor } \\
\text { que incluya la localización, características, aparición } \\
\text { / duración, frecuencia, calidad, intensidad o severidad } \\
\text { del dolor y factores desencadenantes. } \\
\text { 140003. Asegurarse de que el paciente reciba los } \\
\text { cuidados analgésicos correspondientes. } \\
\text { 140007. Determinar el impacto de la experiencia del } \\
\text { dolor sobre la calidad de vida (sueño, apetito, activi- } \\
\text { dad, función cognoscitiva, humor, relaciones, trabajo y } \\
\text { responsabilidad de roles). } \\
\text { 140013. Determinar la frecuencia necesaria para la } \\
\text { realización de una valoración de la comodidad del } \\
\text { paciente y poner en práctica un plan de seguimiento. }\end{array}$ \\
\hline
\end{tabular}




\begin{tabular}{|c|c|c|}
\hline NANDA & NOC & NIC \\
\hline $\begin{array}{l}\text { 00046. Deterioro de la integridad } \\
\text { cutánea. } \\
\text { Características definitorias: } \\
\text { - Alteración de la superficie de la } \\
\text { piel } \\
\text { Factores relacionados: } \\
\text { - Déficit inmunitario }\end{array}$ & $\begin{array}{l}\text { 1101. Integridad tisular: piel y mem- } \\
\text { branas mucosas. } \\
\text { Indicadores: } 110115 \text {. Lesiones cutá- } \\
\text { neas. } \\
\text { Escala Likert } 2 \text { (de gravemente com- } \\
\text { prometido a no comprometido) } \\
\text { Puntuación basal: } 2 \\
\text { Al alta puntuación:5 }\end{array}$ & $\begin{array}{l}\text { 3660. Cuidados de las heridas. } \\
\text { Actividades. } \\
\text { 366003. Monitorizar las características de la herida, } \\
\text { incluyendo drenaje, color, tamaño y olor. } \\
366004 \text {. Medir el lecho de la herida. } \\
366006 \text {. Limpiar/curar la herida según indicación. } \\
366011 \text {. Aplicar el vendaje adecuado al tipo de herida. } \\
\text { 366015. Cambiar el apósito según la cantidad de } \\
\text { exudado y drenaje. } \\
366016 \text {. Inspeccionar la herida cada vez que se reali- } \\
\text { za el cambio de vendaje. } \\
366030 \text {. Documentar la localización, el tamaño y el } \\
\text { aspecto de la herida. } \\
366017 \text {. Comparar y registrar cualquier cambio pro- } \\
\text { ducido en la herida. }\end{array}$ \\
\hline $\begin{array}{l}\text { 00085. Deterioro de la movilidad } \\
\text { física. } \\
\text { Características definitorias: } \\
\text { - Limitación de la amplitud de } \\
\text { movimiento } \\
\text { Factores relacionados: } \\
\text { - Deterioro neuromuscular }\end{array}$ & $\begin{array}{l}\text { 0219. Movimiento articular hombro. } \\
\text { Indicadores: } 21901 \text {. Flexión hacia } \\
\text { adelante de } 180^{\circ} \\
21903 \text {. Extensión de } 50^{\circ} \\
21903 \text {. Rotación externa de } 90 \\
21904 \text {. Rotación interna de } 90^{\circ} \\
21905 \text {. Abducción de } 180^{\circ} \\
21907 \text {. Flexión hacia delante de } 180^{\circ} \\
21910 \text {. Rotación interna de } 90^{\circ} \\
21911 \text {. Abducción de } 180^{\circ} \\
\text { Escala Likert } 2 \text { (de gravemente com- } \\
\text { prometido a no comprometido) } \\
\text { Puntuación basal:4 } \\
\text { Al alta puntuación:5 }\end{array}$ & $\begin{array}{l}\text { 224. Enseñanza: actividad/ejercicios prescritos } \\
\text { Actividades: } \\
\text { 561203. Enseñar al paciente a realizar la actividad/ } \\
\text { ejercicio prescripto } \\
561212 \text {. Enseñar al paciente a realizar un precalenta- } \\
\text { miento y enfriamiento antes y después de la actividad / } \\
\text { ejercicio y la importancia de tal acción.. } \\
\text { 561213. Enseñar al paciente una buena postura y la } \\
\text { mecánica corporal, si procede. } \\
561214 \text {. Observar al paciente mientras realiza la } \\
\text { actividad / ejercicio. } \\
561221 \text {. Incluir a la familia / ser querido, si resulta } \\
\text { apropiado. }\end{array}$ \\
\hline $\begin{array}{l}\text { 00204. Perfusión tisular periférica } \\
\text { ineficaz } \\
\text { Factores relacionados: } \\
\text { Diabetes }\end{array}$ & $\begin{array}{l}\text { 1622. Conducta de cumplimiento: } \\
\text { dieta prescripta } \\
\text { Toma alimentos compatibles con la } \\
\text { dieta prescripta } \\
\text { Bebe líquidos compatibles con la dieta } \\
\text { prescripto } \\
\text { Evita alimentos y líquidos no permitidos } \\
\text { en la dieta. } \\
\text { Escala Likert (de siempre demostrado } \\
\text { a nunca demostrado) } \\
\text { Puntuación basal:4 } \\
\text { Al alta puntuación:5 }\end{array}$ & $\begin{array}{l}\text { 1100. Manejo de la nutrición } \\
\text { 110002. Determinar el estado nutricional del paciente } \\
\text { y su capacidad para satisfacer las necesidades nutri- } \\
\text { cionales } \\
110003 \text {. Determinar las preferencias nutricionales } \\
110004 \text {. Instruir al paciente sobre las necesidades } \\
\text { nutricionales } \\
110007 \text {. Proporcionar la selección de alimentos nece- } \\
\text { sarios se oriente hacia opciones más saludables. }\end{array}$ \\
\hline $\begin{array}{l}\text { 00004. Riesgo de infección } \\
\text { Factores relacionados: } \\
\text { Alteración de las defensas primarias } \\
\text { (rotura de la piel, traumatismo de } \\
\text { los tejidos, disminución de la acción } \\
\text { ciliar, estasis de los líquidos corpora- } \\
\text { les, cambio del pH de las secreciones, } \\
\text { alteración del peristaltismo) }\end{array}$ & $\begin{array}{l}\text { 1902. Control del riesgo } \\
\text { 190201. Reconoce los factores de } \\
\text { riesgo } \\
\text { Escala Likert (de siempre demostrado } \\
\text { a nunca demostrado) } \\
\text { Puntuación basal:4 } \\
\text { Al alta puntuación:5 }\end{array}$ & $\begin{array}{l}\text { 6540. Control de infección } \\
\text { Actividades: } \\
\text { 654001. Distribuir la superficie correspondiente por } \\
\text { paciente, según los consejos de los Centros para el } \\
\text { control de enfermedades (CDC) } \\
\text { 654002. Limpiar el ambiente adecuadamente después } \\
\text { de cada uso por parte de los pacientes. } \\
\text { 654004. Aislar a las personas expuestas a enfermeda- } \\
\text { des transmisibles. } \\
\text { 654006. Limitar el número de visitas } \\
654017 \text {. Fregar la piel del paciente con un agente } \\
\text { antibacteriano. }\end{array}$ \\
\hline
\end{tabular}


- Mejoraron el estado de la integridad cutánea y no adquirieron infección.

- Lograron controlar el dolor.

- La segunda paciente mejoró la movilidad del brazo izquierdo.

\section{Discusión}

Como se ha podido apreciar claramente las pacientes presentaban como patología de base un LES, y acudieron al servicio de urgencias por una recidiva por herpes zoster.

EI LES es una enfermedad de carácter crónico, autoinmune, multisistémica, de etiología desconocida, que se caracteriza por la presencia de anticuerpos y complejos inmunes. Esta enfermedad presenta manifestaciones clínicas diversas, que atacan en sentido general, de forma masiva, aunque fracciona sus ataques en brotes y por sistemas desde el propio inicio de la enfermedad ${ }^{7}$. Las infecciones ocurren tanto al inicio de la enfermedad como en etapas tardías. El herpes zoster es el agente viral más común que afecta a estos pacientes ${ }^{8}$.

En ambos casos los pacientes no presentaban antecedentes de patologías renales presentando un examen cefalocaudal y de laboratorio normal, salvo las patologías de base (hipertensión y diabetes), que tendrían porqué interferir en el desarrollo de nefropatía por depósito de cristales. El tratamiento antiviral fue prescrito correctamente a dosis apropiadas. En el primer caso clínico se observó la progresión de la IRA, al segundo día, en una paciente más joven, mientras que en el segundo caso la progresión ocurrió al octavo día, en una paciente adulta mayor, por lo que la edad no es tomada en esta ocasión como un factor determinante. Tras confirmarse la nefrotoxicidad asociada al tratamiento con Aciclovir se indicó rápidamente la suspensión del medicamento indicado, lo que mejoró en pocas horas el cuadro clínico presentado por las pacientes.

Con respecto a los cuidados de enfermería, estos fueron aplicados y enfocados apropiadamente de forma integral, poniendo especial énfasis en la protección de la función renal, en el cuidado de las lesiones de la piel, el dolor, la educación, la contención y el apoyo emocional de los pacientes.

El cuidado proporcionado por enfermería en ambos casos ha sido integral cubriendo todas las dimensiones de los pacientes, sus cuidados fueron dirigidos tanto a las intervenciones derivadas de los diagnósticos de enfermería como de los problemas surgidos debido al tratamiento aplicado?.
Es fundamental el papel de enfermería en el control de los factores de riesgo que se produce por el desequilibrio electrolítico, ya que pueden poner en peligro la vida del paciente. Poch y Manuell ${ }^{10}$ hablan del manejo agudo del enfermo renal, en el que el tratamiento debe incluir el mejora el estado hemodinámico y volémico del paciente, evitar nuevos problemas renales y optimizar la nutrición.

Una vez confirmada la nefrotoxicidad asociada al Aciclovir en progresión a IRA y tras suspender de forma inmediata el tratamiento antiviral, enfermería debe brindar cuidados a las medidas de soporte indicadas como: control estricto metabólico, restricción hídrica y balance estricto hidroeléctrico, para lograr no sólo la mejoría sino también la resolución del daño renal ${ }^{11}$. Es fundamental el rol profesional que desempeña enfermería en la administración y seguimiento del tratamiento ya que la detección temprana en el pronóstico de la IRA es clave para que el paciente recupere su función renal lo más satisfactoriamente posible, evitando llegar a la necesidad de realizar diálisis o evitando que progrese a una insuficiencia renal crónica ${ }^{11}$.

Se ha encontrado bibliografía que sustenta las complicaciones nefrotóxicas que desarrolla el Aciclovir ${ }^{1,2,5,6,11}$, sin embargo, aunque hemos encontrado algún artículo que menciona la infección de herpes zoster con LES ${ }^{12,13}$, es nula la información encontrada sobre LES con recidiva de herpes zoster en progresión con IRA por toxicidad medicamentosa, por lo que consideramos este artículo podría ser relevante en la información brindada para futuras investigaciones científicas.

En conclusión, es menester para enfermería realizar un seguimiento minucioso de los pacientes que presentan tratamiento con Aciclovir, especialmente de aquellos que presentan de base patologías crónicas, como LES en recidivas con herpes zoster, para prevenir eventos adversos asociados al fármaco.

\section{Agradecimientos}

A la Doctora Valeria Pachioli por su apoyo a enfermería y los Doctores Paloma Dulevich, María Daniela Salinas, María Lía Morales, Jorge Barbieri, Jorge y Alberto Guida por su colaboración.

Recepción: 08-09-20

Aceptación: 15-09-21

Publicación: 30-12-21 


\section{Bibliografía}

1. Vela Enríquez F, Sánchez Vázquez A, Cañabate Reche F, Vicente Pintor A, Benavides Román R. Insuficiencia renal aguda por Aciclovir, ¿un efecto secundario infraestimado? An Pediatr (Barc) [Internet] 2008; [consultado 01 May 2021].69(2):103-245. Disponible en: https://www.analesdepediatria.org/espdf-S1695403308720307.

2. Guerrero C, Moyano J, Ulloa M, Pacheco S, Chinchilla P. Nefropatía por Aciclovir en un paciente con sospecha de toxicidad por opioides: caso clínico. Rev. Soc. Esp. Del Dolor [Internet] 2015 [consultado 01 May 2021].22(1):36-8. Disponible en: http:// scielo.isciii.es/scielo.php?script=sci_arttext\&pi$\mathrm{d}=S 113480462015000100006 \& \operatorname{lng}=\mathrm{es}$ http://dx.doi. org/10.4321/S1134-80462015000100006.

3. Arguedas Quesada J. El riñón y la cinética de los fármacos. Acta Médica Costarricense, Colegio de Médicos y Cirujanos. [Internet] 1997 [consultado 01 May 2021]. Disponible en: http://www.binasss.sa.cr/revistas/amc/v39n2/art5.pdf.

4. Morales J. Drogas Nefrotóxicas. Rev. Med. Clin. Condes. [Internet] 2010 [consultado $28 \mathrm{Abr}$ 2021];21(4):623-8. Disponible en http://www.clinicalascondes.com/area-academica/pdf/MED_21_4/14_ Dr_Morales.pdf.

5. Pacheco L, Tavares H, Neto MM, Dantas M, D'Oliveira Rocha L, Mota Ribeiro K. Insuficiencia renal aguda associada ao uso de Aciclovir endovenoso. Rev Assoc Med Bras. [Internet] 2005 [consultado 01 May 2021];51(5):275-8. Disponible en: http://www.scielo. br/pdf/\%0D/ramb/v5ln5/al9v5ln5. pdf.

6. Machado Alba J, Medina Morales D, Parrado Fajardo I. Insuficiencia renal aguda secundaria a Aciclovir. Reporte de caso y revisión de la literatura. [Internet] 2015 [consultado 28 Abr 2021];19(2):88-91. Disponible en https://www.sciencedirect.com/science/article/pii/S0123939214000472.
7. Sánchez-Rodríguez S. Lupus eritematoso: enfermedad autoinmune sistémica y órgano específica. Rev. Biomédica. [Internet] 2004 [consultado 01 May 2021]; 15(3). Disponible en https://doi.org/10.32776/ revbiomed.v15i3.388.

8. Hernández Muñiz Y, Cruz García Y, Mirabal H. Infección por Herpes Zoster en una paciente con Lupus Eritematoso Sistémico. Rev Cub de Reumatología. [Internet] 2011. [consultado 28 Abr 2021];13:17-8. disponible en: http://www.sld.cu/sitios/reumatologia/ temas.php?idv=23736.

9. Abril Sabater MD, Iglesias Sanjuán R, Jerez Garcia, A, López Parra M J., Mañé Buixó N, Yuste Jiménez E. Aplicación de una técnica cualitativa para la elaboración de un plan de atención al paciente pluripatológico en hemodiálisis. Rev Soc Esp Enferm Nefrol. 2012;7(4):67-71.

10. Liaño García F, Álvarez Rangel LE, Junco E, Rodríguez Palomares JR, Candela Toha A, Cigarrán Guldrís S. Guías SEN. Actuación en el Fracaso Renal Agudo. Nefrología. 2007;27(3):135-9.

11. Fleischer R, Johnson M. Acyclovir nephrotoxicity: a case report highlighting the importance of prevention, detection, and treatment of acyclovir-induced nephropathy. Case Reports in medicine [Internet]. 2010 [consultado $28 \mathrm{Abr} 2021$ ]. Disponible en: https://doi. org/10.1155/2010/602783.

12. Ferreira J, Marques $H$, Ferriani $M$, Gormezano $N$, Terreri $M$, Pereira $R$, et al. Herpes zoster infection in childhood-onset systemic lupus erythematosus patients: a large multicenter study. Lupus [Internet]. 2016 [consultado 03 Sep 2021];25(7):7549. Disponible en: https://pubmed.ncbi.nlm.nih. gov/26821966/.

13. Tsappa I, Missouris C, Psarellis S. Acyclovir-induced thrombocytopaenia in a patient with SLE. BMJ Case Rep [Internet]. 2018 [consultado 03 Sep 2021]. Disponible en: https://www.ncbi.nlm.nih. gov/pmc/articles/PMC6011485/.

Este artículo se distribuye bajo una Licencia Creative Commons Atribución-NoComercial 4.0 Internacional. https://creativecommons.org/licenses/by-nc/4.0/ 